\title{
- NOTES -
}

\section{CANONICAL APPROACH TO BIHARMONIC VARIATIONAL PROBLEMS*}

\author{
BY
}

A. M. ARTHURS

University of York, England

Abstract. A canonical approach to biharmonic variational problems is presented It provides a new form of the principle of stationary energy and a new derivation of the principle of minimum potential energy.

1. Introduction. In a recent series of papers [1]-[4], variational principles associated with the canonical equations

$$
T \Phi=\partial W / \partial U, \quad T^{*} U=\partial W / \partial \Phi
$$

have been studied. The work of Noble [1] dealt with the one-dimensional case $T=$ $d / d x, T^{*}=-d / d x$, while the operators $T=\operatorname{grad}, T^{*}=-\operatorname{div}$ have been discussed in papers on diffusion and related topics [2]-[4].

The purpose of this note is to present some results for the operators $T=\nabla^{2}, T^{*}=\nabla^{2}$. The theory is used to derive a canonical form of the principle of stationary energy for biharmonic problems and to provide a new derivation of the principle of minimum potential energy.

2. Theory. We consider a physical problem which is described by the pair of canonical Euler equations

$$
\begin{aligned}
T \Phi & =\frac{\partial}{\partial U} W(\mathbf{r}, \Phi, U) \\
T^{*} U & =\frac{\partial}{\partial \Phi} W(\mathbf{r}, \Phi, U)
\end{aligned}
$$

in which $T$ and $T^{*}$ are linear operators, $\mathrm{r}$ is a position vector and $\Phi$ and $U$ are functions of $\mathrm{r}$. The region $R$ is a part of the $x y$-plane which has a piecewise smooth boundary $B$. The boundary conditions are taken to be

$$
\begin{aligned}
\Phi & =\varphi_{0} \\
\partial \Phi / \partial n & =f(U) \quad \text { on } \quad B
\end{aligned}
$$

where $n$ is the outward pointing normal to the boundary, and $\varphi_{0}$ and $f$ are given functions. The operator $T^{*}$ in (2) is the adjoint of $T$ in the sense that

$$
(U, T \Phi)=\left\langle T^{*} U, \Phi\right\rangle,
$$

where the inner products are defined by

*Received April 8, 1968. 


$$
\begin{gathered}
(U, T \Phi)=\int_{R} U T \Phi d x d y-\int_{B} U \frac{\partial \Phi}{\partial n} d s+\int_{B} F(U) d s \\
\left\langle T^{*} U, \Phi\right\rangle=\int_{R}\left(T^{*} U\right) \Phi d x d y-\int_{B} \frac{\partial U}{\partial n} \Phi d s+\int_{B} F(U) d s
\end{gathered}
$$

with

$$
F(U)=\int^{U} f\left(U^{\prime}\right) d U^{\prime}
$$

Definitions (6) and (7) are appropriate to the case $T=\nabla^{2}, T^{*}=\nabla^{2}$. If we introduce the functional

$$
I(\Phi, U)=\int_{R} W(\mathbf{r}, \Phi, U) d x d y-(U, T \Phi),
$$

then the following results are obtained:

Stationary property. $I(\Phi, U)$ is stationary at $(\varphi, u)$ if Eqs. (1)-(4) hold simultaneously at $(\varphi, u)$.

Extremum principle. Choose a trial function $\Phi$ which is equal to $\varphi_{0}$ on $B$, and determine $U(\Phi)$ so that Eqs. (1) and (4) are satisfied identically. Then, if (2) holds at $(\varphi, u)$ we have from (9)

$$
G(\Phi) \equiv I(\Phi, U(\Phi))=I(\varphi, u)+\delta^{2} I(\Phi)+O(\Phi-\varphi)^{3},
$$

where

$$
\begin{aligned}
\delta^{2} I(\Phi)=\frac{1}{2} \int_{R}\left\{(\Phi-\varphi)^{2}\left[\frac{\partial^{2} W}{\partial \Phi^{2}}\right]_{\varphi, u}-(U(\Phi)\right. & \left.-u)^{2}\left[\frac{\partial^{2} W}{\partial U^{2}}\right]_{\varphi, u}\right\} d x d y \\
& +\frac{1}{2} \int_{B}(U(\Phi)-u)^{2}\left[\frac{d f}{d U}\right]_{\varphi, u} d s .
\end{aligned}
$$

If terms of third and higher orders can be neglected (or if they vanish), it follows that

$$
G(\Phi) \leq I(\varphi, u) \text { if } \delta^{2} I \leq 0
$$

or

$$
G(\Phi) \geq I(\varphi, u) \quad \text { if } \delta^{2} I \geq 0 .
$$

Thus we have an upper or a lower bound for $I(\varphi, u)$ depending on the sign of $\delta^{2} I$. The pair of functions $(\varphi, u)$ furnishes the exact solution of the problem in Eqs. (1)-(4).

3. The biharmonic equation. The equation which governs the small deflection bending of a thin elastic plate is [5], [6]

$$
\nabla^{4} \Phi=q / D \text { in } \mathrm{R}
$$

where $\Phi$ is the deflection normal to the surface, $q(x, y)$ is the distribution of normal loading and $D$ is the flexural rigidity. The boundary conditions [5], [6] for a plate which is clamped on part $B_{1}$ and simply supported on $B-B_{1}$ are

$$
\Phi=\partial \Phi / \partial n=0 \quad \text { on } B_{1},
$$


and

$$
\Phi=\partial \Phi / \partial n-1 / \kappa(1-\nu) \nabla^{2} \Phi=0 \text { on } B-B_{1} .
$$

Here $\kappa$ is the local curvature of $B$ and $\nu$ is Poisson's ratio. In addition we assume that these deflection conditions satisfy Eq. (14) explicitly. We now write (14) as the pair of equations

$$
\begin{aligned}
\nabla^{2} \Phi & =U \quad \text { in } \quad R . \\
\nabla^{2} U & =q / D
\end{aligned}
$$

This way of writing (14) is equivalent to that used by Morley [5], but the canonical form of (17) and (18) does not seem to have been emphasized previously. The boundary value problem in (15)-(18) is a special case of that discussed in Sec. 2 and corresponds to

$$
\begin{aligned}
& W(\mathbf{r}, \Phi, U)=\frac{1}{2} U^{2}+q \Phi / D, \\
& T=\nabla^{2}, \quad T^{*}=\nabla^{2}, \\
& \varphi_{0}=0 \text { on } B \text {, } \\
& f(U)=0 \quad \text { on } B_{1} \text {, } \\
& =U / \kappa(1-\nu) \text { on } B-B_{1} \text {. }
\end{aligned}
$$

Putting these in (9) we obtain

$$
I(\Phi, U)=\int_{R}\left\{\frac{1}{2} U^{2}+\frac{q}{D} \Phi-U \nabla^{2} \Phi\right\} d x d y+\int_{B-B_{1}}\left\{U \frac{\partial \Phi}{\partial n}-\frac{U^{2}}{2 \kappa(1-\nu)}\right\} d s .
$$

From Sec. 2 we see that $I(\Phi, U)$ in (23) is stationary at $(\varphi, u)$ where $\varphi, u$ are the exact solutions of (15)-(18). This is a canonical form of the principle of stationary energy.

When $\Phi=\varphi$ and $U=u$ we find that (23) gives

$$
I(\varphi, u)=\frac{1}{2} \int_{R} u^{2} d x d y-\frac{1}{2(1-\nu)} \int_{B-B_{1}} \frac{1}{\kappa} u^{2} d s .
$$

The functional $G(\Phi)$ given by (10) is found to be

$G(\Phi)=\int_{R}\left\{-\frac{1}{2}\left(\nabla^{2} \Phi\right)^{2}+\frac{q}{D} \Phi\right\} d x d y+\frac{1}{2} \int_{B-B_{2}} \kappa(1-\nu)\left(\frac{\partial \Phi}{\partial n}\right)^{2} d s, \quad \Phi=0 \quad$ on $B$.

In addition Eq. (11) becomes

$$
\delta^{2} I(\Phi)=-\frac{1}{2} \int_{R}(U(\Phi)-u)^{2} d x d y+\frac{1}{2(1-\nu)} \int_{B-B_{1}} \frac{1}{\kappa}(U(\Phi)-u)^{2} d s .
$$

If

$$
\kappa(1-\nu)<0
$$

then

$$
\delta^{2} I(\Phi) \leq 0
$$

and hence, by (12), we have the variational principle

$$
G(\Phi) \leq I(\varphi, u)
$$

Eq. (29) is equivalent to the principle of minimum potential energy [5], [6]. 
Acknowledgment. I should like to thank Sir H. Jeffreys for valuable comments on this work.

\section{REFERENCES}

[1] B. Noble, Univ. Wisconsin Math. Res. Center Rep. No. 473 (1964)

[2] A. M. Arthurs, Proc. Roy. Soc. Ser. A298, 97 (1967)

[3] A. M. Arthurs and P. D. Robinson, Proc. Roy. Soc. Ser. A303, 497 (1968)

[4] A. M. Arthurs and P. D. Robinson, Proc. Roy. Soc. Ser. A303, 503 (1968)

[5] L. S. D. Morley, Q. J. Mech. Appl. Math. 19, 371 (1966)

[6] S. G. Mikhlin, Variational methods in mathematical physics, Chapter 4, Pergamon Press, London, 1964 